01

\title{
Исследование лазерной фотоионизации ядерного изомера ${ }^{177 m} \mathrm{Lu}$
}

\author{
(ㄷ А.Б. Дьячков, А.А. Горкунов, А.В. Лабозин, К.А. Маковеева, С.М. Миронов, \\ В.Я. Панченко, В.А. Фирсов, Г.О. Цветков Ф
}

Национальный исследовательский центр „Курчатовский институт“, 123182 Москва, Россия

ฯ e-mail: Tsvetkov_GO@nrcki.ru

Поступила в редакцию 22.08.2019 г.

В окончательной редакции 24.09.2019 г.

Принята к публикации 01.10.2019 г.

Впервые методом лазерной резонансной фотоионизационной спектроскопии исследована сверхтонкая структура переходов трехступенчатой схемы фотоионизации лютеция $5 d 6 s^{2}{ }^{2} D_{3 / 2}-5 d 6 s 6 p^{4} F_{5 / 2}^{o}$ $5 d 6 s 7 s^{4} D_{3 / 2}-\left(53375 \mathrm{~cm}^{-1}\right)_{1 / 2}^{o}$ для ядерного изомера ${ }^{177 m} \mathrm{Lu}$. В результате определены константы сверхтонкого расщепления и энергии уровней, а также магнитный дипольный и электрический квадрупольный моменты ядра ${ }^{177 m} \mathrm{Lu}$. Исследованы изотопические сдвиги спектральных линий изомера по отношению к природным изотопам и ${ }^{177} \mathrm{Lu}$.

Ключевые слова: изотопический сдвиг, сверхтонкая структура, квадрупольный момент.

DOI: $10.21883 /$ OS.2020.01.48832.251-19

\section{Введение}

Метод получения радиофармпрепарата ${ }^{177} \mathrm{Lu}$ (период полураспада 6.7 суток), предложенный в работе [1], основан на селективной лазерной фотоионизации изотопов ${ }^{177} \mathrm{Lu}$, получаемых искусственно путем нейтронного облучения в ядерном реакторе природного лютеция $\left({ }^{175} \mathrm{Lu}-97.4 \%\right.$ и $\left.{ }^{176} \mathrm{Lu}-2.6 \%\right)$. В зависимости от длительности облучения и интенсивности нейтронного потока содержание ${ }^{177} \mathrm{Lu}$ составляет $10^{-5}-10^{-3}$. При этом важно отметить, что приблизительно $10^{-3}$ всех атомов ${ }^{177} \mathrm{Lu}$ являются долгоживущими изомерами ${ }^{177 m} \mathrm{Lu}$ [2]. Ядро изомера находится в возбужденном высокоспиновом состоянии $(E=0.97 \mathrm{MeV}$, спин ядра $I=23 / 2)$ с периодом полураспада $\tau \approx 160$ суток. Распад происходит в основном посредством $\beta$-перехода в ${ }^{177} \mathrm{Hf}(78 \%)$, остальное приходится на $\gamma$-переходы и внутреннюю конверсию. При лечении пациентов изотопы лютеция выводятся из организма, но не могут быть утилизированы госпиталями как обычные отходы и должны длительное время $(>>10 \tau)$ содержаться в отдельных хранилищах из-за продолжительной радиационной активности ${ }^{177 m} \mathrm{Lu}$. Мы предполагаем, что лазерная фотоионизация ${ }^{177} \mathrm{Lu}$ по схеме $5 d 6 s^{2}{ }^{2} D_{3 / 2}-5 d 6 s 6 p^{4} F_{5 / 2}^{o}$ $5 d 6 s 7 s^{4} D_{3 / 2}-\left(53375 \mathrm{~cm}^{-1}\right)_{1 / 2}^{o}$ может эффективно исключить из процесса долгоживущий изомер ${ }^{177 m} \mathrm{Lu}$, снизить его долю в конечном продукте и, как следствие, повысить конкурентоспособность данного медицинского препарата.

Селективность фотоионизации изотопов ${ }^{177} \mathrm{Lu}$ достигается благодаря изотопическим сдвигам отдельных линий сверхтонких структур (СТС) изотопов лютеция. СТС переходов для ${ }^{175} \mathrm{Lu},{ }^{176} \mathrm{Lu},{ }^{177} \mathrm{Lu}$, изотопические сдвиги уровней, селективность фотоионизации ${ }^{177} \mathrm{Lu}$ исследовалась нами в работах $[1,3,4]$. К сожалению, малое содержание $\left(<10^{-7}\right)$ изомера не позволило исследовать его сверхтонкую структуру. Тем не менее, по крайней мере, на первой ступени фотоионизации возможны большие частотные сдвиги СТС линий ${ }^{177 m} \mathrm{Lu}$ относительно ${ }^{177} \mathrm{Lu}$, что позволит селективно (по длине волны) отстраиваться от возбуждения и вовлечения в процесс ионизации атомов нецелевого изомера. Так, для двух из пяти селективных каналов ионизации ${ }^{177} \mathrm{Lu}$ частотные расстояния до ближайших линий изомера на первой ступени составили $\sim 600 \mathrm{MHz}$ и $1700 \mathrm{MHz}$ [3]. Из-за различий в СТC ${ }^{177} \mathrm{Lu}$ и ${ }^{177 m} \mathrm{Lu}$ другие каналы ионизации также могут оказаться селективными за счет переходов второй и/или третьей ступени.

В данной работе экспериментально исследовалась сверхтонкая структура ${ }^{177 m} \mathrm{Lu}$ на всех ступенях схемы фотоионизации $5 d 6 s^{2}{ }^{2} D_{3 / 2}-5 d 6 s 6 p^{4} F_{5 / 2}^{o}-5 d 6 s 7 s^{4} D_{3 / 2}$ $\left(53375 \mathrm{~cm}^{-1}\right)_{1 / 2}^{o}$. Очевидно, что знание СТС позволяет оценить возможности селективной фотоионизации и самого изомера. Дело в том, что в последнее время в мире нарастает активность, направленная на создание генератора ${ }^{177} \mathrm{Lu}$, основанного на переходе метастабильного ${ }^{177 m} \mathrm{Lu}$ в основное состояние [5]. В частности, сформулировано предложение использовать ${ }^{177 m} \mathrm{Lu}$, связанный хелатором DOTA на сорбенте. Распад ${ }^{177 m} \mathrm{Lu}$, сопровождающийся переходом в основное состояние ${ }^{177} \mathrm{Lu}$, приводит к селективной диссоциации последнего вследствие эффекта Сциларда-Чалмерса. В результате раствор, промывающий сорбент, может содержать ${ }^{177} \mathrm{Lu}$ с высокой концентрацией и быть использован для терапии. Такой генератор дает возможность независимо от облучения в реакторе длительное время снабжать лечебное учреждение радионуклидом. Создание такого генератора может потребовать обогащения облученного лютеция по ${ }^{177 m} \mathrm{Lu}$, которое может быть осуществлено лазерным фотоионизационным методом. 


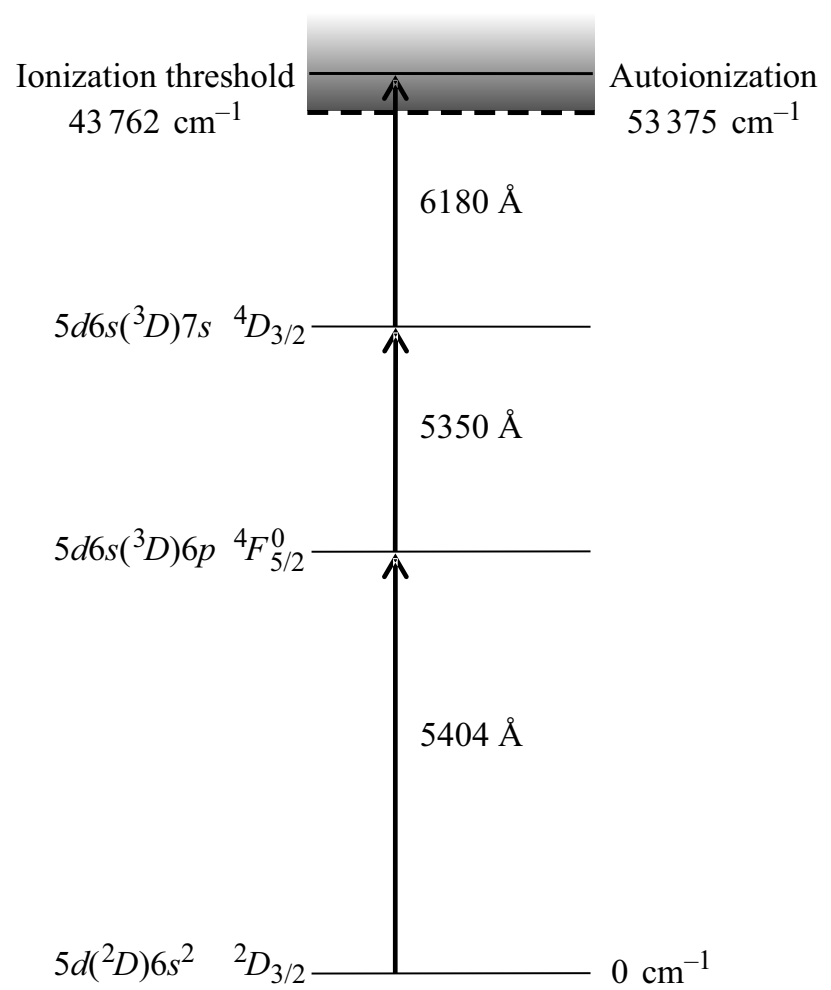

Рис. 1. Схема трехступенчатой лазерной фотоионизации LuI.

\section{Эксперимент}

Исследования сверхтонкой структуры $177 m \mathrm{Lu}$ на всех ступенях схемы фотоионизации $5 d 6 s^{2}{ }^{2} D_{3 / 2}$ $5 d 6 s 6 p^{4} F_{5 / 2}^{o}-5 d 6 s 7 s^{4} D_{3 / 2}-\left(53375 \mathrm{~cm}^{-1}\right)_{1 / 2}^{o}$ (рис. 1) проводились методом лазерной резонансной ионизационной масс-спектроскопии (LRIMS). Для резонансного возбуждения и ионизации атомов использовалось излучение трех импульсных одномодовых лазеров на красителях со спектральной шириной линии генерации $100-150 \mathrm{MHz}$ (FWHM), накачиваемых лазерами на парах меди. Для регистрации фотоионов использовался коммерческий квадрупольный масс-спектрометр МС-7302. Технические параметры и особенности экспериментальной установки детально описаны в работах $[1,3]$.

В отличие от традиционной масс-спектрометрии, когда изотопы с разными атомными весами регистрируются отдельно, атомные массы ${ }^{177} \mathrm{Lu}$ и ${ }^{177 m} \mathrm{Lu}$ практически одинаковы. Различие составляет $\sim 0.001 \mathrm{amu}$ $(\sim 0.97 \mathrm{MeV}[6])$, что недоступно для разрешения квадрупольным масс-спектрометром. Поэтому в эксперименте могли быть зарегистрированы только те линии CTC изомера ${ }^{177 m} \mathrm{Lu}$, частоты которых более чем на $100 \mathrm{MHz}$ отличались от линий основного изотопа ${ }^{177} \mathrm{Lu}$, определенных в работе [3].

Изомер ${ }^{177 m} \mathrm{Lu}$ был получен в результате облучения $0.6 \mathrm{~g}$ металлического лютеция на реакторе ИР-8 в потоке нейтронов $1.4 \cdot 10^{13} \mathrm{~cm}^{-2} \mathrm{~s}^{-1}$. Образец облучался в течение 49 дней, после чего выдерживался два месяца. К этому моменту количество ${ }^{177} \mathrm{Lu}$ и ${ }^{177 m} \mathrm{Lu}$ в образце стало приблизительно одинаковым. Активность и соотношение радионуклидов ${ }^{177} \mathrm{Lu}$ и ${ }^{177 m} \mathrm{Lu}$ определялись методом $\gamma$-спектрометрии. Для измерений использовался гамма-спектрометр Canberra GL0515R с детектором из сверхчистого германия. Обработка спектров и анализ радионуклидного состава проводились в программе GRANIT с библиотекой констант, сформированной на основе базы рекомендованных данных NUDAT (МАГАTЭ). Радионуклид ${ }^{177} \mathrm{Lu}$ определяли по линиям $112.95 \mathrm{keV}(6.4 \%)$ и $208.37 \mathrm{keV}$ (11.0\%), эти линии имеются также и у ${ }^{177 m} \mathrm{Lu}$, однако в данном случае его вкладом можно пренебречь из-за малого количества и существенно более длительного периода полураспада (160.44 суток). ${ }^{177 m} \mathrm{Lu}$ определяли по линиям $378.5 \mathrm{keV}$ $(29.9 \%), 413.7 \mathrm{keV}(17.5 \%)$. К началу эксперимента концентрация ${ }^{177} \mathrm{Lu}$ составляла $(3.4 \pm 0.4) \cdot 10^{-6}$.

\section{Результаты и обсуждение}

Так как изотопы ${ }^{175} \mathrm{Lu},{ }^{176} \mathrm{Lu},{ }^{177} \mathrm{Lu},{ }^{177 m} \mathrm{Lu}$ имеют ненулевой спин ядра, все уровни энергии (рис. 1) расщеплены в мультиплеты. Количество подуровней в мультиплете определяется полным атомным моментом атома $F=J+I, J+I-1, \ldots,|J-I|$, где $J-$ полный электронный момент, $I-$ спин ядра. Спектр СТС составляют переходы между подуровнями мультиплетов с изменением момента $\Delta F=0 ; \pm 1$, в результате чего весь спектр схемы фотоионизации ${ }^{177 m} \mathrm{Lu}$ состоял из 30 линий СТС (рис. 2).

В ходе исследования СТС первого перехода $5 d 6 s^{22} D_{3 / 2}-5 d 6 s 6 p^{4} F_{5 / 2}^{o}$ лазер первой ступени сканировался по длине волны в окрестности перехода. Лазеры второй и третьей ступеней стабилизировались на расчетных значениях длин волн, соответствующих определенному каналу фотоионизации изомера с одного из подуровней $F=9-14$ уровня $5 d 6 s 6 p^{4} F_{5 / 2}^{o}$. Прогнозные значения длин волн всех линий были предварительно извлечены по константам сверхтонкого расщепления уровней ${ }^{177 m} \mathrm{Lu}$, энергиям уровней (центров тяжести) ${ }^{175} \mathrm{Lu}$ и расчетным значениям изотопических сдвигов между ${ }^{177 m} \mathrm{Lu}$ и ${ }^{175} \mathrm{Lu}$ [3]. Предложенный в работе способ расчета изотопического сдвига для изомера на первой ступени был применен для оценки сдвигов последующих уровней $5 d 6 s 7 s^{4} D_{3 / 2}$ и $\left(53375 \mathrm{~cm}^{-1}\right)_{1 / 2}^{o}$. В зависимости от верхнего подуровня регистрировалось от одного до трех ионизационных резонансов. Результат сканирования с ионизацией через верхний подуровень $F=12$ представлен на рис. 3. На рис. 4 результат сканирования с одновременной регистрацией линии $5 \rightarrow 6$ основного изотопа ${ }^{177} \mathrm{Lu}$. Для того чтобы избежать влияния двухфотонных процессов на точность определения длины волны, импульсы лазеров второй и третьей ступеней были задержаны относительно первой ступени на величину длительности импульса ( 20ns). Время жизни уровня 


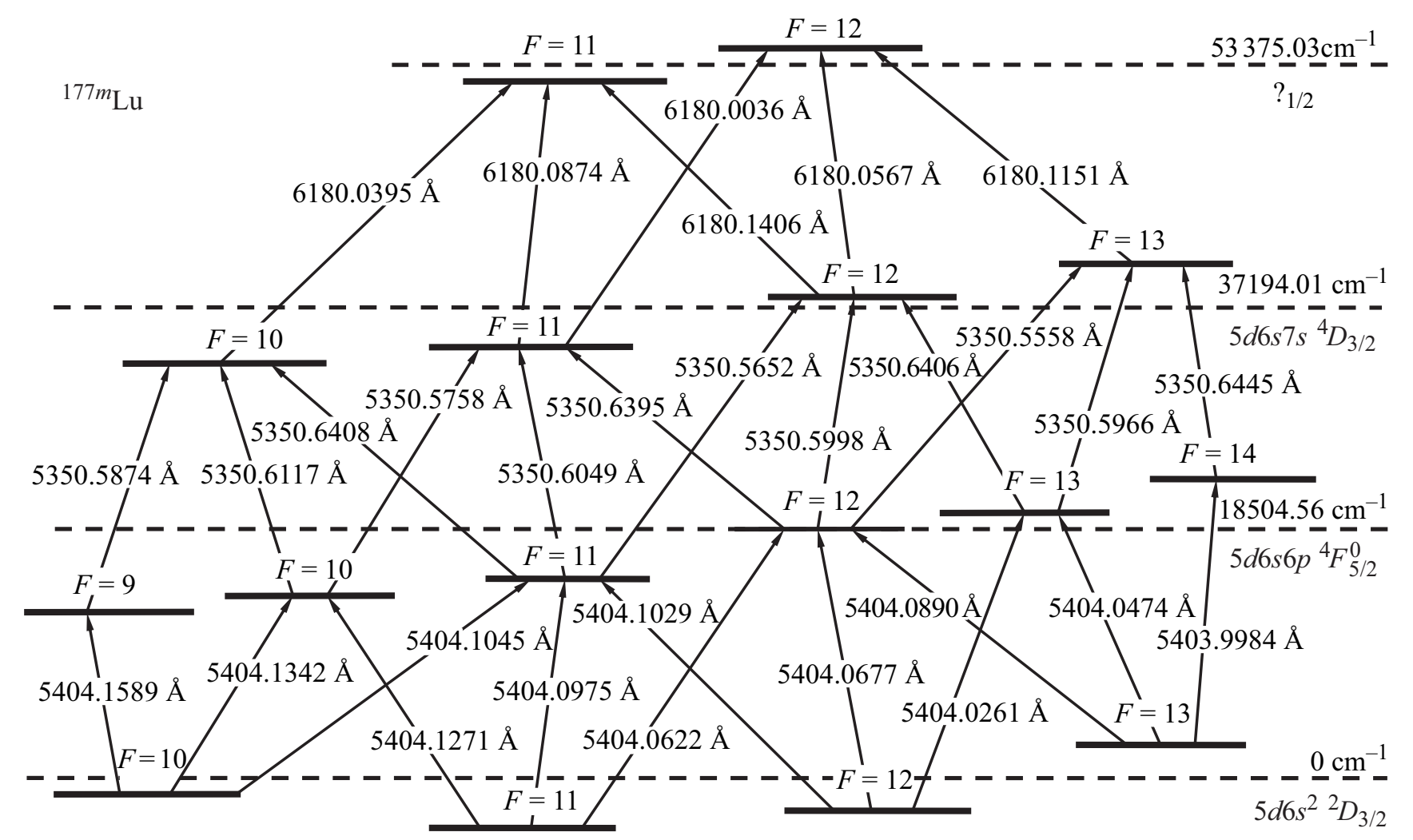

Рис. 2. Каналы фотоионизации ${ }^{177 m} \mathrm{Lu}$ через компоненты сверхтонкой структуры. Терм верхнего возбужденного автоионизационного уровня $53375.03 \mathrm{~cm}^{-1}$ не определен.

$5 d 6 s 6 p^{4} F_{5 / 2}^{o}$ составляет $472 \mathrm{~ns}$ [7], поэтому по окончании импульса почти все резонансно возбужденные атомы участвовали в дальнейшей ионизации. Интенсивности в лучах 2-й и 3-й ступеней значительно превышали уровни насыщения соответствующих переходов $\left(10 \mathrm{~mW} / \mathrm{cm}^{2}\right.$ и $\left.5 \mathrm{~W} / \mathrm{cm}^{2}\right)$. Это позволило сохранять эффективную ионизацию при отклонении расчетных длин волн второй и третьей ступеней от истинных значений переходов в пределах нескольких $\mathrm{GHz}$. В результате спектрально разрешены были 11 из 12 линий сверхтонкой структуры ${ }^{177 m} \mathrm{Lu}$ первого перехода. Одна из линий $10 \rightarrow 9$ в пределах $30 \mathrm{MHz}$ совпала по частоте с линией $2 \rightarrow 2$ основного изотопа ${ }^{177} \mathrm{Lu}$ и не была разрешена.

Исследование СТС второго и третьего переходов проводилось в разведенных во времени импульсах (импульсы лазера третьей ступени были дополнительно задержаны на $20 \mathrm{~ns}$ ). Лазеры первых ступеней стабилизировались на длинах волн отдельных СТС линий, значения которых были определены на предыдущих стадиях эксперимента. Спектрально были разрешены все линии 2-го и 3-го переходов (12 и 6 соответственно). Для точного нахождения „центра“ каждой линии (длины волны) экспериментальные точки фотоионизационных резонансов аппроксимировались контуром Фойгта с использованием метода наименьших квадратов. Значения

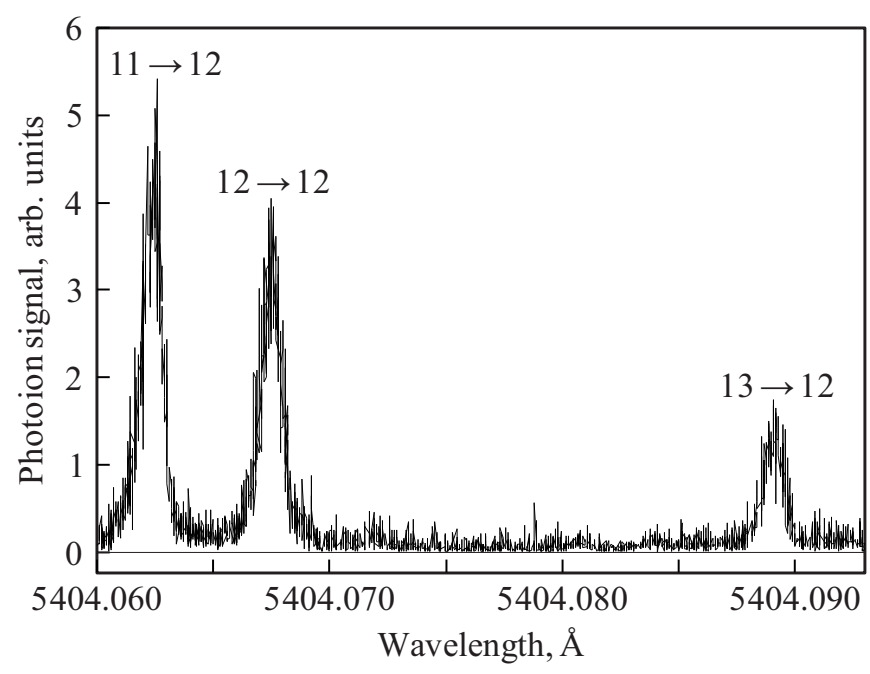

Рис. 3. Фотоионный сигнал ${ }^{177 m} \mathrm{Lu}$ при сканировании длины волны лазера первой ступени при $\lambda_{2}(12 \rightarrow 13)=5350.5557 \AA$ и $\lambda_{3}(13 \rightarrow 12)=6180.1155 \AA$ (расчетные значения).

длин волн всех линий СТС приведены на общей схеме фотоионизации ${ }^{177 m} \mathrm{Lu}$, рис. 2.

Сверхтонкое расщепление атомных уровней (энергетическое смещение подуровней относительно центра тяжести $\Delta_{F}$ ) описывается с помощью констант магнитного 
Таблица 1. Энергия, изотопические сдвиги и константы сверхтонкого расщепления уровней схемы фотоионизации для ${ }^{177 m} \mathrm{Lu}$

\begin{tabular}{c|c|c|c|c|c}
\hline Уровень & Энергия, $\mathrm{cm}^{-1}$ & $A, \mathrm{MHz}$ & $B, \mathrm{MHz}$ & \multicolumn{2}{|c|}{$\begin{array}{c}\text { Изотопический сдвиг } \\
\text { относительно }{ }^{175} \mathrm{Lu}, \mathrm{MHz}\end{array}$} \\
\hline $5 d 6 s^{2}{ }^{2} D_{3 / 2}$ & 0 & $61.2(3)$ & $2472(11)$ & 0 & Ссылки \\
$5 d 6 s 6 p^{4} F_{5 / 2}^{o}$ & $18504.5593(20)$ & $310.5(8)$ & $1840(63)$ & $-812(25)$ & настоящая работа \\
$5 d 6 s 7 s^{4} D_{3 / 2}$ & $37194.0082(27)$ & $348(2)$ & $134(68)$ & $-360(57)$ & настоящая работа \\
$\left(53375 \mathrm{~cm}^{-1}\right)_{1 / 2}^{o}$ & $53375.0324(43)$ & $549(6)$ & 0 & $-507(120)$ & настоящая работа \\
\end{tabular}

Таблица 2. Магнитный дипольный и электрический квадрупольный моменты ядра ${ }^{177 m} \mathrm{Lu}$ и результаты других работ [13]

\begin{tabular}{c|c|c|c}
\hline $\begin{array}{c}\text { Магнитный дипольный } \\
\text { момент, } \mu_{N}\end{array}$ & $\begin{array}{c}\text { Электрический (спектроскопический) } \\
\text { квадрупольный момент, barn }\end{array}$ & Ссылка, год & Метод измерения \\
\hline $2.74(21)$ & & {$[14], 1974$} & $\begin{array}{c}\text { Гамма-детектирование } \\
\text { с ориентированными ядрами }\end{array}$ \\
\hline $3.3(3)$ & $4.23(67)$ & {$[15], 1975$} & $\begin{array}{c}\text { Гамма-детектирование } \\
\text { с ориентированными ядрами }\end{array}$ \\
\hline $2.337(13)$ & $5.2(5)$ & {$[16], 1983$} & $\begin{array}{c}\text { Гамма-детектирование } \\
\text { с ориентированными ядрами }\end{array}$ \\
\hline $2.308(11)$ & $5.71(5)$ & {$[17], 1996$} & ЯМР с ориентированными ядрами \\
\hline $2.31(3)$ & $5.69(12)$ & {$[9], 1998$} & Лазерная коллинеарная спектроскопия \\
\hline & & эта работа & Лазерная резонансная \\
\end{tabular}

дипольного $(A)$, электрического квадрупольного $(B)$ взаимодействий и зависит от $J, I$ и $F[8]$ :

$$
\Delta_{F}=\frac{A}{2} K+\frac{B}{4} \frac{\frac{3}{2} K(K+1)-2 I(I+1) J(J+1)}{I J(2 I-1)(2 J-1)},
$$

где $K=F(F+1)-J(J+1)-I(I+1)$. Для каждой линии СТС конкретного перехода можно записать:

$$
\Delta E+\Delta_{F^{\prime}}-\Delta_{F}-1 / \lambda_{F F^{\prime}}=0,
$$

где $\Delta E\left(\mathrm{~cm}^{-1}\right)$ - энергия между центрами тяжести уровней перехода, $\lambda_{F F^{\prime}}(\mathrm{cm})$ - длина волны линии $F \rightarrow F^{\prime}$. Система (1) была решена относительно $A, B$ и $\Delta E$ по всем зарегистрированным линиям (длинам волн) посредством $\chi^{2}-$ оптимизации для каждой ступени. Результаты представлены в табл. 1. Погрешность измерений констант и изотопических сдвигов уровней определялась погрешностью в определении длин волн линий СТС относительно друг друга, которая во всех экспериментах не превысила значение $\pm 0.0003 \AA$. Погрешность в определении абсолютных значений энергии уровней включала абсолютную ошибку измерений длин волн прибором LM-007 (Laser 2000, GmbH): $\pm 0.0005 \AA$.

Отметим, что для нахождения констант основного состояния $5 d 6 s^{2} D_{3 / 2}$ использовалась несколько иная процедура, рассматривались только переходы из основного состояния на один возбужденный подуровень.

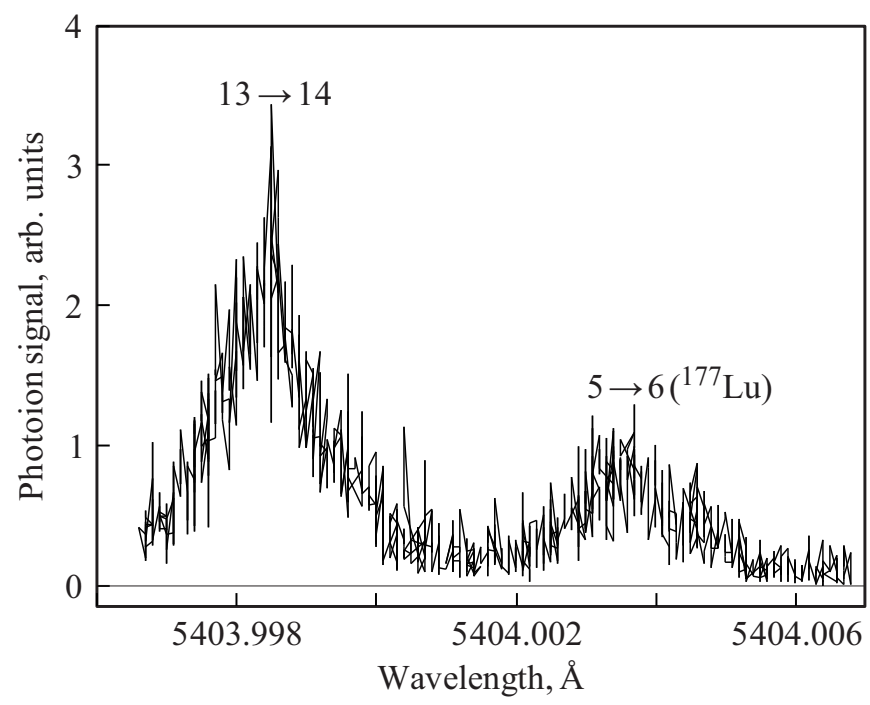

Pис. 4. Фотоионный сигнал ${ }^{177 m} \mathrm{Lu}$ и ${ }^{177} \mathrm{Lu}$ при сканировании длины волны лазера первой ступени при $\lambda_{2}(14 \rightarrow 13)=5350.6444 \AA$ и $\lambda_{3}(13 \rightarrow 12)=6180.1155 \AA($ pacчетные значения).

Это позволило исключить при обработке параметры возбужденного уровня и повысить точность определения констант основного состояния, что было необходимо для определения ядерного магнитного момента ${ }^{177 m} \mathrm{Lu}$. Для 
Таблица 3. Селективные каналы ионизации ${ }^{177} \mathrm{Lu}$ (по компонентам CTC) [3] и ближайшие по частотам каналы возбуждения ${ }^{177 m} \mathrm{Lu}$ со сдвигами частот по ступеням

\begin{tabular}{c|c|c}
\hline $\begin{array}{c}\text { Канал ионизации } \\
{ }_{177} \mathrm{Lu}, \mathrm{F}-\mathrm{F}\end{array}$ & $\begin{array}{c}\text { Ближайший по частоте } \\
\text { канал ионизации }{ }^{177 m} \mathrm{Lu}, \mathrm{F}-\mathrm{F}\end{array}$ & $\begin{array}{c}\text { Сдвиг частоты }\left(\text { относительно }{ }^{177} \mathrm{Lu}\right) \\
\text { по ступеням возбуждения, MHz }\end{array}$ \\
\hline $5-6-5-4$ & $13-14-13-12$ & $+575 ;-377 ;-23$ \\
$5-5-5-4$ & $12-12-13-12$ & $-40 ;+2387 ;-23$ \\
$4-3-4-3$ & $10-10-11-11$ & $-226 ;-2827 ;+3650$ \\
$2-3-4-3$ & $10-10-11-11$ & $-100 ;-2827 ;+3650$ \\
$2-1-2-3$ & $10-9-10-11$ & $+1675 ;-630 ;-300$
\end{tabular}

Таблица 4. Селективные каналы ионизации ${ }^{177 m} \mathrm{Lu}$ (по компонентам СТC). Для каждой схемы указаны ближайшие по частотам каналы возбуждения изотопов ${ }^{175} \mathrm{Lu},{ }^{176} \mathrm{Lu},{ }^{177} \mathrm{Lu}$ и частотные расстояния до ${ }^{177 m} \mathrm{Lu}$ на всех ступенях возбуждения

\begin{tabular}{c|c|c|c}
\hline \multirow{2}{*}{$\begin{array}{c}\text { Канал ионизации } \\
{ }_{177 m} \mathrm{Lu}, \mathrm{F}-\mathrm{F}\end{array}$} & \multicolumn{2}{|c}{ Сдвиг частоты (относительно $\left.{ }^{177 m} \mathrm{Lu}\right)$ по ступеням возбуждения, МНz } \\
\cline { 2 - 4 } & ${ }^{175} \mathrm{Lu}$ & ${ }^{176} \mathrm{Lu}$ & ${ }^{177} \mathrm{Lu}$ \\
\hline \multirow{2}{*}{$12-13-13-12$} & $4-5-5-4$ & $8.5-8.5-8.5-7.5$ & $4-5-5-4$ \\
& $-996 ;+1267 ;-237$ & $-1110 ;+995 ;-932$ & $-2167 ;+1885 ;+24$ \\
\hline \multirow{2}{*}{$13-13-13-12$} & $5-5-5-4$ & $8.5-8.5-8.5-7.5$ & $4-5-5-4$ \\
& $-852 ;+1267 ;+237$ & $+1078 ;+995 ;-932$ & $+20 ;+1885 ;+24$ \\
\hline $12-12-13-12$ & $5-5-5-4$ & $6.5-7.5-8.5-7.5$ & $5-5-5-4$ \\
& $+1232 ;-3005 ;+237$ & $+298 ;+2858 ;-932$ & $+40 ;-2387 ;+24$ \\
\hline \multirow{2}{*}{$10-10-10-11$} & $3-2-2-3$ & $7.5-6.5-6.5-6.5$ & $2-3-2-3$ \\
& $-832 ;+880 ;+545$ & $+1274 ;+1225 ;-3640$ & $+103 ;-1110 ;+298$
\end{tabular}

любой пары изотопов 1 и 2 выполняется равенство:

$$
\frac{{ }^{1} A}{{ }^{2} A}=\frac{1 g_{I}}{{ }^{2} g_{I}}\left(1+{ }^{1} \Delta^{2}\right)
$$

где $g_{I}$ - ядерное гиромагнитное отношение: $g_{I}=\mu_{I} / I$ $\left(\mu_{I}-\right.$ магнитный дипольный момент ядра со спином I). ${ }^{1} \Delta^{2}$ - разница сверхтонких аномалий для данных изотопов, учитывающая различия в пространственных распределениях магнитного момента. Соотношение (2) в приближении ${ }^{1} \Delta^{2}=0$ было использовано для извлечения магнитного момента ядpa ${ }^{177 m} \mathrm{Lu}$ по константам магнитного расщепления основного уровня. В качестве референтного был выбран изотоп ${ }^{175} \mathrm{Lu}(I=7 / 2)$, для которого значения магнитного момента $\mu_{I}\left({ }^{175} \mathrm{Lu}\right)=2.2323(11) \mu_{N} \quad$ и $A^{175}\left(5 d 6 s^{2}{ }^{2} D_{3 / 2}\right)=194.33162(9) \mathrm{MHz}$ определены с высокой точностью методом ядерного магнитного резонанса [10]. Следует отметить, что разница сверхтонких аномалий ${ }^{1} \Delta^{2}$, как правило, не превышает $10^{-3}$ [11] и соответственно не влияет на результат измерений, находясь в пределах экспериментальной ошибки. Аналогичным образом был извлечен электрический квадрупольный момента изомера: $Q^{(1)} / B^{(1)}=Q^{(2)} / B^{(2)}$. Здесь референтными были выбраны $Q\left({ }^{175} \mathrm{Lu}\right)=3.49(2)$ barn из [12] и $B^{175}\left(5 d 6 s^{2}{ }^{2} D_{3 / 2}\right)=1511.39865(69) \mathrm{MHz}$ [10]. Полученные значения моментов ядра ${ }^{177 m} \mathrm{Lu}$ (табл. 2) хорошо совпали с результатами из последних работ $[9,17]$.
Исходя из спектра сверхтонкой структуры ${ }^{177 m} \mathrm{Lu}$, для 5 селективных каналов фотоионизации ${ }^{177} \mathrm{Lu}$ [3] были определены ближайшие по частоте каналы ионизации изомера и частотные расстояния по каждой ступени возбуждения, табл. 3. Оказалось, что для всех каналов, как минимум, на одной ступени возбуждения существует большой изотопический сдвиг $(600-3000 \mathrm{MHz})$, соответственно фотоионизация ${ }^{177} \mathrm{Lu}$ останется селективной и по отношению к изомеру.

С точки зрения проведения селективной фотоионизации самого изомера было проведено сравнение СТС спектров ${ }^{177 m} \mathrm{Lu} \mathrm{и}{ }^{175} \mathrm{Lu},{ }^{176} \mathrm{Lu},{ }^{177} \mathrm{Lu} \mathrm{[3].} \mathrm{В} \mathrm{результате}$ было найдено 4 канала с наибольшими сдвигами частот по отношению к ближайшим каналам ионизации природных изотопов лютеция, табл. 4 (селективность по ${ }^{177} \mathrm{Lu}$ может проявляться со временем за счет его быстрого распада).

Ранее на каждом из пяти наиболее селективных каналов фотоионизации ${ }^{177} \mathrm{Lu}$ была получена селективность $\geq 10^{5}$ [4]. При этом световые интенсивности по ступеням были близки к уровням насыщения, а импульсы лазеров второй и третьей ступеней задержаны относительно импульса первой ступени на время длительности импульса. Следует отметить, что в этой ситуации частотный сдвиг на первой ступени является определяющим для селективности. В случае фотоионизации ${ }^{177 m} \mathrm{Lu}$ частотные расстояния по первой ступени 
оказались в среднем на 20\% меньше соответствующих сдвигов в исследованных каналах ${ }^{177} \mathrm{Lu}$. Исходя из этого при наличии больших сдвигов на второй и/или третьей ступенях можно с уверенностью утверждать, что в схожих световых условиях селективность лазерной фотоионизации ${ }^{177 m} \mathrm{Lu}$ составит $10^{4}-10^{5}$.

\section{Выводы}

При разработке лазерного фотоионизационного метода получения радионуклида ${ }^{177} \mathrm{Lu}$ для применения в медицине важным является вопрос о селективности фотоионизации ${ }^{177} \mathrm{Lu}$ по отношению к изомеру ${ }^{177 m} \mathrm{Lu} \mathrm{c}$ точки зрения очистки радионуклида от долгоживущего изомера. Впервые методом лазерной фотоионизационной спектроскопии проведено исследование сверхтонкой структуры изомера на переходах, входящих в состав трехступенчатой схемы фотоионизации $5 d 6 s^{2}{ }^{2} D_{3 / 2}$ $5 d 6 s 6 p^{4} F_{5 / 2}^{o}-5 d 6 s 7 s^{4} D_{3 / 2}-\left(53375 \mathrm{~cm}^{-1}\right)_{1 / 2}^{o}$. Определены константы сверхтонкого расщепления данных уровней, их энергии, изотопические сдвиги, а также магнитный дипольный и электрический квадрупольный моменты ядра ${ }^{177 m} \mathrm{Lu}$. Результаты исследований показали значительные отличия в спектрах поглощения ${ }^{177} \mathrm{Lu}$ и изомера ${ }^{177 m} \mathrm{Lu}$, позволяющие достигать высокую селективность фотоионизации ${ }^{177} \mathrm{Lu} \mathrm{c}$ использованием спектрально узкого (< $150 \mathrm{MHz})$ лазерного излучения. Обнаруженные различия в спектрах ${ }^{177 m} \mathrm{Lu}$ и природных изотопов ${ }^{175} \mathrm{Lu}$ и ${ }^{176} \mathrm{Lu}$ позволили спрогнозировать высокий уровень селективности $\left(10^{4}-10^{5}\right)$ лазерной фотоионизации самого изомера, что может оказаться весьма перспективным для целей ядерной медицины.

\section{Благодарности}

Авторы выражают благодарность С.С. Арзуманову, Ю.Н. Панину, Ю.В. Вязовецкому, А.В. Курочкину и Д.Ю. Чувилину за помощь в подготовке образца ${ }^{177 m} \mathrm{Lu}$.

\section{Финансирование работы}

Исследование выполнено за счет гранта Российского научного фонда (проект № 17-13-01180).

\section{Конфликт интересов}

Авторы заявляют, что у них нет конфликта интересов.

\section{Список литературы}

[1] D'yachkov A.B., Firsov V.A., Gorkunov A.A., Labozin A.V., Mironov S.M., Panchenko V.Y., Semenov A.N., Shatalova G.G., Tsvetkov G.O. // Appl. Phys. B. 2015. V. 121. N 4. P. 425. doi 10.1007/s00340-015-6248-0

[2] Henkelmann R., Hey A., Buck O. // Physics for Health in Europe. 2010. Contribution (CERN).
[3] D'yachkov A.B., Gorkunov A.A., Labozin A.V., Mironov S.M., Tsvetkov G.O., Panchenko V.Y., Firsov V.A. // Opt. Spectrosc. 2018. V. 125. N 6. P. 839. doi 10.1134/S0030400X19020127

[4] Ageeva I.V., D'yachkov A.B., Gorkunov A.A., Labozin A.V., Mironov S.M., Panchenko V.Y., Firsov V.A., Tsvetkov G.O., Tsvetkova E.G. // Quantum Electron. 2019. V. 49. N 9. P. 832. doi 10.1070/QEL17049

[5] Bhardwaj R., Van Der Meer A., Das S.K., De Bruin M., Gascon J., Wolterbeek H.T., Denkova A.G., Serra-Crespo P. // Sci. Rep. 2017. V. 7. 44242. doi 10.1038/srep44242

[6] NuDat 2.7 Электронный ресурс. Режим доступа: nndc.bnl.gov/nudat2

[7] Fedchak J.A., Den Hartog E.A., Lawler J.E., Palmeri P., Quinet P., Biemont E. // Astrophys. J. 2000. V. 542. N 2. P. 1109. doi $10.1086 / 317034$

[8] Woodgate G.K. // Elementary Atomic Structure. 2nd ed. Oxford University Press. 1989.

[9] Georg U., Borchers W., Keim M., Klein A., Lievens P., Neugart R., Neuroth M., Rao P.M., Schulz C. // Eur. Phys. J. A. 1998. V. 3. N 3. P. 225. doi $10.1007 / \mathrm{s} 100500050172$

[10] Brenner T., Büttgenbach S., Rupprecht W., Träber F. // Nucl. Phys. A. 1985. V. 440. N 3. P. 407. doi 10.1016/03759474(85)90237-4

[11] Persson J.R. // Atomic Data and Nuclear Data Tables. 2013. V. 99. P. 62

[12] Dey W., Ebersold P., Leisi H.J., Scheck F., Walter H.K., Zehnder A. // Nucl. Phys. A. 1979. V. 326. N 2-3. P. 418. doi 10.1016/0375-9474(79)90401-9

[13] Stone N.J. // 2014. Table of Nuclear Magnetic Dipole and Electric Quadrupole Moments (INDC(NDS)-0658). International Atomic Energy Agency (IAEA).

[14] Krane K.S., Olsen C.E., Steyert W.A. // Phys. Rev. C. 1974. V. 10. N 2. P. 825. doi 10.1103/PhysRevC.10.825

[15] Schoeters E., Silverans R.E., Vanneste L., Freitag K., Hübel H. // Z. Physik. A. 1975. V. 272. N 2. P. 203. doi 10.1007/BF01408148

[16] Oertel D., Kettschau A., Brewer W.D., Vanneste L., Leuven U. // Z. Physik. A. 1983. V. 310. N 3. P. 233. doi 10.1007/BF01415228

[17] König C., Hagn E., Hinfurtner B., Zech E., Eder R. // Phys. Rev. C. 1996. V. 54. N 3. P. 1027. doi $10.1103 /$ physrevc.54.1027 